\title{
PATH PLANNING FOR MULTIPLE FEATURES BASED LOCALIZATION
}

\author{
Francis Celeste, Frederic Dambreville \\ Dept. EORD FAS Team, DGA ,16 bis av. Prieur de la cte d'Or, 94114 Arcueil, France \\ francis.celeste@etca.fr,frederic.dambreville@etca.fr \\ Jean-Pierre Le Cadre \\ IRISA/CNRS, Campus de Beaulieu, 35042 Rennes, France \\ lecadre@irisa.fr
}

Keywords: $\quad$ path planning, Cramèr Rao Bound, map-based localization, dynamic programming.

\begin{abstract}
In surveillance or exploration mission in a known environment, the localization of the dedicated sensor is of main importance. In this paper, we discuss the path planning problem for the localization algorithm which correlates range and bearing measurements and a map composed of several features. The sensor motion is designed from an information measure derived from the Fisher Information Matrix. It is shown that a closed form expression of the cost can be obtained. The optimal features location can be neatly geometrically interpreted. An integral cost which includes the sensor perception limitation is then formulated. It is used in a dynamic programming framework to tackle the path optimization problem.
\end{abstract}

\section{INTRODUCTION}

The path planning problem for map-based localization consists in designing the best trajectory for a mobile in a known environment, which guarantees the highest performance of positioning during its execution. Data collected from sensors are "matched" to a prior map to estimate the state (e.g., position and heading). Depending on the sensor dynamic and the observation models, different localization algorithms can be used. When the system is linear or near linear with Gaussian noises, Kalman-based approaches are relevant (Thrun et al., 2005; S. Thrun and Dellaert, 2000). In this paper, we introduce a framework to compute "optimal" path for a moving vehicle which collects range and bearing data from $2 \mathrm{D}$ features. One of the main challenges is to choose an appropriate measure to be optimized. In random estimation, the Fisher Information Matrix (FIM) can be used. We considered a D-optimal design (Paris and Le Cadre, 2002). The first interesting result of this work is the derivation of a closed form expression for the FIM determinant. It is shown that it depends on groups of two or three features. Then, a geomet- ric analysis of the optimal features placement can be done. By exploiting this measure, we introduce an integral cost functional for a path space, which is composed of elementary moves with constant velocity and constant heading. Moreover, the sensor field of view limitations are included to the cost computation. At last, we formulate the problem as finding an optimal path on a graph by means of dynamic programming. The paper ends with one illustrative example.

\section{PROBLEM FORMULATION}

We consider a moving sensor evolving according to the dynamic model

$$
\begin{aligned}
\dot{x}_{t} & =v_{t} \cos \varphi_{t}, \\
\dot{y}_{t} & =v_{t} \sin \varphi_{t}, \\
\dot{\varphi}_{t} & =\omega_{t} .
\end{aligned}
$$

where its state $\mathbf{X}_{t} \triangleq\left[x_{t}, y_{t}, \varphi_{t}\right]$ is composed of its 2 -D position and its orientation. A feature map of its environment is available for localization purpose. In equation 2 , we assume that the known 
control $\mathbf{u}_{t} \triangleq\left[v_{t}, \omega_{t}\right] \in \mathcal{U} \subset \mathbb{R}^{2}$. During its displacement, the mobile gets sensor measurements from detected features which are in the embedded map. Let us denote $\mathbf{f}_{t} \triangleq\left\{f_{1}, \ldots, f_{m_{t}}\right\}$ the set of $m_{t}$ features visible and used in the localization process at time $t$. Each feature is defined by its 2D position in a global frame $\mathcal{R}_{g} \triangleq(O, \vec{u}, \vec{v})$ :

$$
f_{i} \leftrightarrow\left(x^{i}, y^{i}\right) \in \mathcal{D} \subset \mathbb{R}^{2} .
$$

and the "sensor-feature" vector $\delta p_{i}(t) \triangleq$ $\left[x^{i}-x_{t}, y^{i}-y_{t}\right]^{*}$. The measurements vector is the stacked vector $\mathbf{Z}_{t}=\left[z_{1}^{t}, \ldots, z_{m_{t}}^{t}\right]$ where $z_{i}^{t}$ is the range and bearing measurement for feature $f_{i}$. So, the observation model stands as follows :

$$
\mathbf{Z}_{t}=\mathbf{H}_{\mathbf{t}}\left(\mathbf{X}_{t}, \mathbf{f}_{t}\right)+\mathbf{W}_{\mathbf{t}} .
$$

where the $2 \times \mathrm{i}^{\text {th }}$ and $2 \times \mathrm{i}+1^{\text {th }}$ elements of $\mathbf{H}_{\mathbf{t}}\left(\mathbf{X}_{t}, \mathbf{f}_{t}\right)$ are the components of the two dimensional vector $h\left(\mathbf{X}_{t}, f_{i}\right)$ given by

$$
\begin{gathered}
z_{i}^{t}=h\left(\mathbf{X}_{t}, f_{i}\right)+w_{t}^{i} . \\
h\left(\mathbf{X}_{t}, f_{i}\right) \triangleq\left\{\begin{array}{l}
\sqrt{\left(x_{t}-x^{i}\right)^{2}+\left(y_{t}-y^{i}\right)^{2}} \\
\operatorname{atan}_{2}\left(\frac{y^{i}-y_{t}}{x^{i}-x_{t}}\right)-\varphi_{t}
\end{array}\right.
\end{gathered}
$$

The noise vector $w_{t}^{i}$ is modelled by an i.i.d. Gaussian process with zero mean and covariance ma$\operatorname{trix} \Sigma_{t}^{i}$. Moreover, we suppose that $\Sigma_{t}^{i}=\Sigma, \forall i$ and

$$
\Sigma=\left(\begin{array}{cc}
\sigma_{r}^{2} & 0 \\
0 & \sigma_{\varphi}^{2}
\end{array}\right) .
$$

We also consider that $w_{t}^{j}$ and $w_{t}^{l}$ are independent for $l \neq j$. So in light of (2), the likelihood function is given by

$$
p\left(\mathbf{Z}_{t} \mid \mathbf{X}_{t}\right) \propto \exp \left(-\frac{1}{2} \sum_{l=1}^{m_{t}}\left\|z_{l}-h\left(\mathbf{X}_{t}, f_{l}\right)\right\|_{\Sigma}^{2}\right) .
$$

If $\hat{\mathbf{X}}_{t}$ is one estimate based on the measurement $\mathbf{Z}_{t}$ (e.g., the maximum likelihood estimate), the covariance error $e_{\mathbf{X}_{t}}=\mathbf{X}_{t}-\hat{\mathbf{X}}_{t}$ is lower bounded by the Cramer Rao Bound (CRB) (Van Trees, 1968).

$$
\operatorname{Cov}\left(e \mathbf{X}_{t}\right) \succ F^{-1}(t) .
$$

The calculation of the FIM $F(t)$ is given in our case by,

$$
F=\sum_{i=1}^{m_{t}}\left(\frac{\partial h\left(\mathbf{X}_{t}, f_{i}\right)}{\partial \mathbf{X}_{t}}\right)^{*} \Sigma^{-1}\left(\frac{\partial h\left(\mathbf{X}_{t}, f_{i}\right)}{\partial \mathbf{X}_{t}}\right) .
$$

The elementary gradient vector can be derived straightforwardly

$$
\frac{\partial h\left(\mathbf{X}_{t}, f_{i}\right)}{\partial \mathbf{X}_{t}}=\left(\begin{array}{ccc}
c_{i} & s_{i} & 0 \\
-\frac{s_{i}}{\rho_{i}} & \frac{c_{i}}{\rho_{i}} & -1
\end{array}\right) .
$$

where $\alpha_{i}(t) \triangleq \angle \vec{u} \delta p_{i}(t), \rho_{i} \triangleq\left\|\delta p_{i}(t)\right\|, c_{i} \triangleq$ $\cos \alpha_{i}$ and $s_{i} \triangleq \sin \alpha_{i}$. Let us also introduce the following notations :

- $\vec{c} \triangleq\left[c_{1} \cdots c_{m_{t}}\right]^{*}, \vec{s} \triangleq\left[s_{1} \cdots s_{m_{t}}\right]^{*}$,

- $\vec{c}_{\rho} \triangleq\left[\frac{c_{1}}{\rho_{1}} \cdots \frac{c_{m_{t}}}{\rho_{m_{t}}}\right]^{*}, \vec{s}_{\rho} \triangleq\left[\frac{s_{1}}{\rho_{1}} \cdots \frac{s_{m_{t}}}{\rho_{m_{t}}}\right]^{*}$.

- $1_{m_{t}} \triangleq[1 \cdots 1]^{*}, 0_{m_{t}} \triangleq[0 \cdots 0]^{*}$

Without loss of generality, we set $\sigma_{d}=\sigma_{\varphi}=1$ then we can rewrite ${ }^{1}$

$$
F(t)=G(t) G(t)^{*} .
$$

with

$$
G(t)=\left(\begin{array}{cc}
\vec{c}^{t} & \overbrace{\overrightarrow{s_{\rho}}}^{t} \\
\vec{s}^{t} & -\overrightarrow{\vec{c}_{\rho}} \\
0_{m_{t}} & 1_{m_{t}}
\end{array}\right) .
$$

$G(t)$ is a $3 \times 2 \mathrm{~m}_{\mathrm{t}}$ matrix with columns $G_{i}$ are part of the subset $\mathcal{G}_{1}(t)$ or $\mathcal{G}_{2}(t)$ :

$$
\begin{aligned}
& \mathcal{G}_{1}(t)=\left\{G_{i_{1}}, 1 \leq i_{1} \leq m_{t} \mid G_{i_{1}}=\left(\begin{array}{lll}
c_{i_{1}} & s_{i_{1}} & 0
\end{array}\right)^{*}\right\}, \\
& \mathcal{G}_{2}(t)=\left\{G_{i_{2}}, 1 \leq i_{2} \leq m_{t} \mid G_{i_{2}}=\left(\begin{array}{lll}
\frac{s_{i_{2}}}{\rho_{i_{2}}} & -\frac{c_{i_{2}}}{\rho_{i_{2}}} & 1
\end{array}\right)^{*}\right\} .
\end{aligned}
$$

In this paper, we are dealing with the optimization of the sequence of displacement which provides the "best" estimate of the state. This can be achieved using an appropriate measure of information gain. We adopt here a D-optimal design considering the determinant of the $\mathrm{FIM}^{2}$. In the next section, we show that this measure is a function implying the estimated bearings angles $\left(\alpha_{i}(t)\right)_{i=1}^{m_{t}}$ and relative ranges $\left(\rho_{i}(t)\right)_{i=1}^{m_{t}}$.

\section{DERIVATION OF $\operatorname{det}(F)$}

Let us define $\mathcal{L}(t)$ as the determinant of the FIM at time $t$ in position $\mathbf{X}_{t}$. From (11), we have

$$
\mathcal{L}(t)=\operatorname{det}\left(G(t) G(t)^{*}\right) .
$$

Using the Binet-Cauchy formula ${ }^{3}$, we can notice that

$$
\mathcal{L}(t)=\sum_{1 \leq i<j<k \leq 2 m_{t}}\left\{\operatorname{det}\left(G_{i}, G_{j}, G_{k}\right)\right\}^{2} .
$$

\footnotetext{
$1 *$ is the transpose operator

${ }^{2}$ other matrix operator can be used, such as the trace

${ }^{3} \operatorname{det}(A B)=\sum_{S} \operatorname{det}\left(A_{s}\right) \operatorname{det}\left(B_{s}\right), S=\{1, \cdots, n\}$, if $A \in \mathcal{M}_{\mathbb{K}}(m, n)$ et $B \in \mathcal{M}_{\mathbb{K}}(n, m), A_{s}$ is the $m \times n$ matrix whose columns are those of $A$ with in $S$
} 
hence to compute $\mathcal{L}(t)$, we have to enumerate the different cases in accordance with the column vectors $\left(G_{i}, G_{j}, G_{k}\right)$ are in $\mathcal{G}_{1}$ or $\mathcal{G}_{2}$. In the following, we denote $d_{i j k} \triangleq \operatorname{det}\left(G_{i}, G_{j}, G_{k}\right)$. If all columns are in $\mathcal{G}_{1}, d_{i j k}$ is trivially equal to zero. Using determinant computation properties and relations betweeen trigonometric functions, we get

a) $G_{i}, G_{j} \in \mathcal{G}_{1}$ and $G_{k} \in \mathcal{G}_{2}$

$$
d_{i j k}^{1}=\sin \left(\alpha_{i}-\alpha_{j}\right) .
$$

b) $G_{i} \in \mathcal{G}_{1}$ and $G_{k}, G_{j} \in \mathcal{G}_{2}$

$$
d_{i j k}^{2}=\frac{\cos \left(\alpha_{i}-\alpha_{k}\right)}{\rho_{k}}-\frac{\cos \left(\alpha_{i}-\alpha_{j}\right)}{\rho_{j}} .
$$

c) $G_{i} \in \mathcal{G}_{1}, G_{j}$ and $G_{k} \in \mathcal{G}_{2}$

$$
d_{i j k}^{3}=\frac{\sin \left(\alpha_{i}-\alpha_{k}\right)}{\rho_{i} \rho_{k}}+\frac{\sin \left(\alpha_{i}-\alpha_{j}\right)}{\rho_{i} \rho_{j}}+\frac{\sin \left(\alpha_{j}-\alpha_{k}\right)}{\rho_{j} \rho_{k}} .
$$

In conclusion, we notice that $\mathcal{L}(t)$ is the sum of three terms $\mathcal{L}_{1}(t), \mathcal{L}_{2}(t)$ and $\mathcal{L}_{3}(t)$ which characterize interactions between pairs and triplets of visible features.

$$
\mathcal{L}(t)=a_{1} \mathcal{L}_{1}(t)+a_{2} \mathcal{L}_{2}(t)+a_{3} \mathcal{L}_{3}(t) .
$$

with $\mathcal{L}_{1}(t)=\sum_{i=1}^{m_{t}} \sum_{j>i}^{m_{t}} g_{1}\left(f_{i}, f_{j}\right), \quad \mathcal{L}_{2}(t)=$ $\sum_{i=1}^{m_{t}} \sum_{j=1}^{m_{t}} \sum_{k>j}^{m_{t}} g_{2}\left(f_{i}, f_{j}, f_{k}\right)$ and $\mathcal{L}_{3}(t)=$ $\sum_{i=1}^{m_{t}} \sum_{j>i}^{m_{t}} \sum_{k>j}^{m_{t}} g_{3}\left(f_{i}, f_{j}, f_{k}\right)$ where $\left(g_{l}\right)_{l \in\{1,2,3\}}$ are respectively given by the square of $d_{i j k}^{l}$ in the above cases. Coefficients $\left(a_{l}\right)_{1 \leq l \leq 3}$ depend on $\sigma_{r}$ and $\sigma_{\varphi}$.

\section{THE OPTIMAL PLACEMENT OF THE FEATURES}

We now study the location of the features which provides the best performance of estimation around a given mean state $\overline{\mathbf{X}}$. The analysis takes into account the sensor field of view and only consider $\mathcal{L}_{1}(t)$ (pairs interaction). Such an approximation is valid when $\frac{\sigma_{d}}{\rho} \ll \sigma_{\varphi}$. Let $\left(f_{i}\right)_{1<i<n}$ be visible from state $\overline{\mathbf{X}}$. We introduce $P=\overline{(x} ; \bar{y}),\left(\overrightarrow{v_{i}}\right)_{1 \leq i \leq n}, D_{m}, \overrightarrow{v_{-}}$and $\overrightarrow{v_{+}}$(see figure 1). $D_{m}$ is the angular aperture of the sensor field of view. An analogy can be made with the reasoning in (Gu et al., 2006) for multiple UAVs cooperation for sensing. The derivation made here is nevertheless simpler and more geometrically intuitive.

Proposition 1 Maximizing $\mathcal{L}_{1}(t)$ is equivalent to find the configuration $\left({\overrightarrow{v_{1}}}^{*}, \ldots, \vec{v}_{n}{ }^{*}\right)$ which minimizes $\left\|\overrightarrow{v_{T}}\right\|=\left\|\sum_{i=1}^{n} \overrightarrow{v_{i}}\right\|^{2}$.

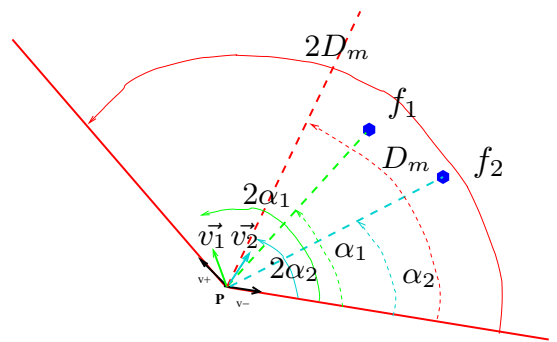

Figure 1: sensor features spatial configuration.

Indeed, using classic trigonometric properties ${ }^{4}$ we can show that $\mathcal{L}_{1}=\frac{1}{4}\left(1-\left\|\sum_{i=1}^{n} \overrightarrow{v_{i}}\right\|^{2}\right)$.

\subsection{Optimal placement for $D_{m}<\frac{\pi}{2}$}

In this context, the value of the angle made by vectors $\overrightarrow{v_{i}}$ and $\overrightarrow{v_{j}}$ is strictly smaller than $\pi$. So $\left\|\overrightarrow{v_{T}}\right\|>0$. Let $i_{0} \in\{1, \cdots, n\}$ and $\theta_{i_{0}}=\angle \overrightarrow{v_{-}} \overrightarrow{v_{0}}$. We also denote $\overrightarrow{v_{\underline{i_{0}}}} \triangleq \sum_{j \neq i_{0}} \overrightarrow{v_{j}}$ and $\theta_{\underline{i_{0}}}=\angle \overrightarrow{v_{-}} \underline{\overrightarrow{v_{0}}}$

$$
\begin{aligned}
\left\|\overrightarrow{v_{T}}\right\|^{2} & =\left\|\overrightarrow{v_{0}}+\overrightarrow{v_{\underline{i_{0}}}}\right\|^{2} \\
& =1+\left\|\underline{\overrightarrow{i_{0}}}\right\|^{2}+2\left\|\overrightarrow{v_{\underline{i_{0}}}}\right\| \cos \left(\theta_{\underline{i_{0}}}-\theta_{i_{0}}\right) .
\end{aligned}
$$

As $D_{m}<\frac{\pi}{2}, \overrightarrow{v_{\underline{i_{0}}}}$ is also between $\overrightarrow{v_{-}}$and $\overrightarrow{v_{+}}$. So, for a given placement of vectors $\left\{\vec{v}_{i}\right\}_{i \neq i_{0}}$, $\left\|\overrightarrow{v_{T}}\right\|$ is minimized for $\theta_{i_{0}}^{*}$ which makes $g\left(\theta_{i_{0}}\right)=$ $\cos \left(\theta_{\underline{i_{0}}}-\theta_{i_{0}}\right)$ minimum.

Proposition 2 In the optimal configuration, each vector $\overrightarrow{v_{i}}$ is on the frontier of the visibility cone.

Proof. $0 \leq \theta_{\underline{i}}, \theta_{i_{0}} \leq 2 D_{m} \Rightarrow \theta_{\underline{i}_{0}}-2 D_{m} \leq \theta_{\underline{i_{0}}}-$ $\theta_{i_{0}} \leq \theta_{\underline{i_{0}}}$. Moreover, $\theta_{i_{0}}-2 D_{m}{ }^{-}>-\pi$ et $\theta_{\underline{i_{0}}} \overline{<} \pi$. We can easily deduce that

$$
\theta_{i_{0}}^{*}=\left\{\begin{array}{ll}
2 D_{m} & \text { if }\left|\theta_{\underline{i_{0}}}-2 D_{m}\right|>\theta_{i_{0}} \\
0 & \text { if }\left|\underline{\theta_{\underline{i_{0}}}}-2 D_{m}\right|<\theta_{\underline{i_{0}}}
\end{array} .\right.
$$

which proves that either $\overrightarrow{v_{0}}=\overrightarrow{v_{-}}$or $\overrightarrow{v_{i_{0}}}=\overrightarrow{v_{+}}$. Let us denote $n_{-}$and $n_{+}$the number of vectors $\overrightarrow{v_{i}}$ respectively equal to $\overrightarrow{v_{-}}$and $\overrightarrow{v_{+}}\left(n_{-}+n_{+}=n\right)$. $n_{-}$must verify the relation

$$
\left\|\overrightarrow{v_{T}}\right\|^{2}=2(1-a) n_{-}^{2}-2(1-a) n n_{-}+n^{2} \triangleq f\left(n_{-}\right) .
$$

with $a=\cos \left(2 D_{m}\right)(a<1) . \quad f$ is minimal for $n_{-}=\frac{n}{2}$, so

- if $n$ is even, $n_{-}=n_{+}=\frac{n}{2}$ and which provides

$$
\mathcal{L}_{1}=\frac{n^{2}}{4} \sin ^{2}\left(D_{m}\right) \text {. }
$$

\footnotetext{
${ }^{4} \sin ^{2} a=\frac{1}{2}(1-\cos 2 a)$ and $\cos (a-b)=\cos a \cos b+$ $\sin a \sin b$
} 
- else we can set $n_{-}=\frac{n-1}{2}$ and $n_{+}=\frac{n+1}{2}$, then $\mathcal{L}_{1}=\frac{n^{2}-1}{4} \sin ^{2}\left(D_{m}\right)$

\subsection{Optimal placement for $D_{m}>\frac{\pi}{2}$}

In this case, we have to make a different reasoning according to the parity of $n$. When $n$ is even, the optimal solution is obvious as we can place the features so that $\overrightarrow{v_{T}}=\overrightarrow{0}$. Indeed, it is enough to choose $\left\{\overrightarrow{v_{1}}, \cdots, \overrightarrow{v_{n}}\right\}$ pairwise such that their difference angle is equal to $\pi$ (i.e. orthogonal assignment of features). We can notice that, there are plenty of such configurations and the cost is $\mathcal{L}_{1}=\frac{n^{2}}{4}$. Otherwise, if $n$ is odd, it is more difficult to find a placement which gives $\overrightarrow{v_{T}}=\overrightarrow{0}$. Nevertheless, we can search among a particular class of configurations with $\overrightarrow{v_{0}}=-\overrightarrow{v_{i_{0}}}$. Assuming $i_{0}=n$, one way to obtain $\overrightarrow{v_{n}}$ collinear and opposite to $\overrightarrow{v_{n}}$, is to choose $\left\{\overrightarrow{v_{1}}, \cdots, \overrightarrow{v_{n-1}}\right\}$ where

$\exists \varphi \in] \frac{\pi}{2}, \pi\left[ \begin{cases}\angle \overrightarrow{v_{i}} \overrightarrow{v_{n}}=\varphi, & \forall i \in\left\{1, \cdots, \frac{n-1}{2}\right\}, \\ \angle \overrightarrow{v_{n}} \overrightarrow{v_{j_{i}}}=\varphi, & \forall j_{i}=i+\frac{n-1}{2} .\end{cases}\right.$

Given $\angle \overrightarrow{v_{-}} \overrightarrow{v_{i}}=\theta_{p}, \forall i \in\left\{1, \cdots, \frac{n-1}{2}\right\}$ and supposing $\overrightarrow{v_{-}}=\vec{u}$, then

$$
\begin{aligned}
\overrightarrow{v_{n}} & =\cos \left(\varphi+\theta_{p}\right) \vec{u}+\sin \left(\varphi+\theta_{p}\right) \vec{v}, \\
\overrightarrow{v_{i}} & =\cos \left(\theta_{p}\right) \vec{u}+\sin \left(\theta_{p}\right) \vec{v}, \forall i, \\
\overrightarrow{v_{i}} & =\cos \left(2 \varphi+\theta_{p}\right) \vec{u}+\sin \left(2 \varphi+\theta_{p}\right) \vec{v}, \forall j_{i} .
\end{aligned}
$$

and $\forall i \in\left\{1, \cdots, \frac{n-1}{2}\right\}$

$$
\begin{aligned}
\overrightarrow{v_{i}}+\overrightarrow{v_{j_{i}}=} & \cos \left(\theta_{p}\right)+\cos \left(2 \varphi+\theta_{p}\right) \vec{u} \\
& +\sin \left(\theta_{p}\right)+\sin \left(2 \varphi+\theta_{p}\right) \vec{v}
\end{aligned}
$$

Using trigonometric properties, we get that:

$$
\begin{aligned}
\overrightarrow{v_{i}}+\overrightarrow{v_{j_{i}}} & =2 \cos (\varphi)\left(\cos \left(\varphi+\theta_{p}\right) \vec{u}+\sin \left(\varphi+\theta_{p}\right) \vec{v}\right) \\
& =2 \cos (\varphi) \overrightarrow{v_{n}} .
\end{aligned}
$$

To make $\overrightarrow{v_{T}}=\overrightarrow{0}$, we must force

$$
\overrightarrow{v_{n}}+\sum_{i=1}^{\frac{n-1}{2}} \overrightarrow{v_{i}}+\overrightarrow{v_{j_{i}}}=\overrightarrow{0}
$$

which is equivalent to the following condition on $\varphi$.

$$
l(\varphi) \triangleq 1+(n-1) \cos (\varphi)=0, \varphi \in\left[\frac{\pi}{2}, \pi[.\right.
$$

As the field of view is limited, we have to satisfy $\varphi \leq D_{m}$. Therefore, if such an angle exists, the cost value is again $\mathcal{L}_{1}=\frac{n^{2}}{4}$. In particular, if
$D_{m}>\frac{2 \pi}{3}$, we can always find an optimal placement. Indeed, it is sufficient to choose $n-3$ vectors as in the even case (orthogonal assignment) and to use the last three with $\varphi=\frac{2 \pi}{3}$. When exists $\varphi$ solution of (17) with $D_{m}<\varphi<\frac{2 \pi}{3}$, it seems difficult to find a configuration which allows to attain the maximum cost. But, we propose a suboptimal solution which minimizes $l(\varphi)$. $l$ is decreasing on $\left[\frac{\pi}{2}, D_{m}\right]\left(\frac{\partial l}{\partial \varphi} \propto-\sin (\varphi)<0\right)$ so its maximum is given for $\varphi=D_{m}$. This leads to the cost value

$$
\mathcal{L}_{1}=\frac{1}{4}\left(n^{2}-\left(1+(n-1) \cos \left(D_{m}\right)\right)^{2}\right) \text {. }
$$

In this section, we made a geometric analysis to determine the optimal placement of the features to maximize the cost $\mathcal{L}_{1}$. Making the same kind of reasoning for the complete cost $\mathcal{L}(t)$ is much more challenging. After this static analysis, we deals with the path planning problem in the next section. For the sake of brevity, we only detail the approach for $\mathcal{L}_{1}(t)$ but it can be generalized to $\mathcal{L}_{2}(t)$ and $\mathcal{L}_{3}(t)$.

\section{PATH PLANNING}

We consider the evolution of the sensor between $\left[t_{0}, t_{f}\right]$ with $0<t_{f} \leq T *$ from position $q_{s} \in \mathcal{D}$ to position $q_{t} \in \mathcal{D}$. We look for paths $\left(\mathbf{X}_{t}\right)_{t \in\left[t_{0}, t_{f}\right]}$ which maximizes the cost

$$
\Psi\left(\left[t_{0}, t_{f}\right]\right)=\int_{t_{0}}^{t_{f}} \mathcal{L}_{1}(t) \mathrm{dt} .
$$

The problem can be formalize in the optimal control framework with two boundaries constraints. Unfortunately, due to the cost expression and the sensor field of view (FOV) limitations, no analytic formulation of the optimal path can be derived. An approximated approach based on the discretization of the state and control space seems more tractable.

\subsection{Path description}

As in (Celeste et al., 2007), We formalize here the problem as a discrete path planning. A regular grid is considered and one path is a sequence of elementary displacements with constant heading $\left(\varphi \in\left\{\varphi_{i}=\frac{i * \pi}{4}, i \in\{-3, \ldots, 4\}\right\}\right)$ and constant velocity $v$ (a leg). For a path $\tau$ with $n_{\tau}$ legs, the cost is as follows:

$$
\Psi\left(\left[t_{0}, t_{f}\right]\right)=\sum_{i=0}^{n_{\tau}-1} \int_{t_{i}}^{t_{i+1}} \mathcal{L}_{1}(t) \mathrm{dt} .
$$


$\mathbf{X}_{t_{0}}=q_{s}$ and $\mathbf{X}_{t_{n_{\tau}-1}}=q_{t}$ are supposed to be on the grid. Some constraints on the maneuvers can be imposed to avoid chaotic behavior (e.g. bang-bang effect)(Paris and Le Cadre, 2002). To solve the planning task we need to compute the cost associated with each leg. First of all, it is necessary to determine the part of the leg where each feature is visible due to the sensor FOV.

\subsection{Cost for one leg}

For a FOV model with an aperture $2 \Delta$ and a maximum range detection $R_{d}$, the area $\mathcal{Z}$ visible from the leg $e$ is composed of three regions $\mathcal{Z}_{1}, \mathcal{Z}_{2}$ and $\mathcal{Z}_{3}$ (see Figure 3 ). A pair of features $\left(f_{i}, f_{j}\right) \in \mathcal{Z}^{2}$ are visible from $P_{-}^{i j}\left(x_{-}^{i j}, y_{-}^{i j}\right)$ and $P_{+}^{i j}\left(x_{+}^{i j}, y_{+}^{i j}\right)$. These limits can be derived using a simple geometric reasoning. Moreover, we have

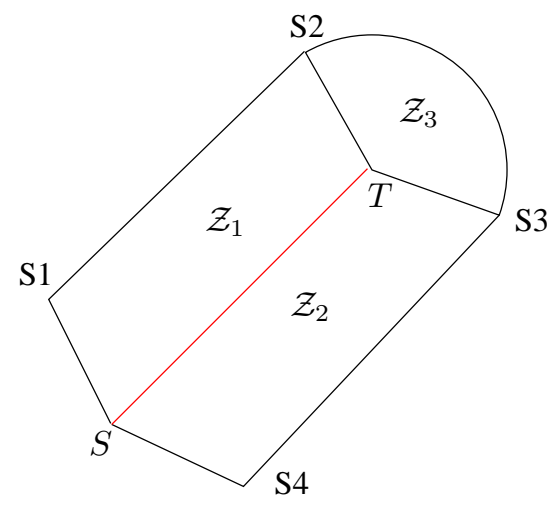

Figure 2: The visible region for one leg.

a relation between an elementary displacement and the associated duration ( $\mathrm{dt} \propto \mathrm{dx}$ if $\varphi \neq \frac{\pi}{2}[\pi]$, $\mathrm{dt} \propto$ dy else). and the leg can be reparametrized as follows:

- $y(x)=\beta+\gamma x, \forall x \in\left[x_{S}, x_{T}\right]$ if $\varphi \neq \frac{\pi}{2}[\pi]$ (non vertical motion),

- $x=x_{S}, y_{S} \leq y \leq y_{T}$ else (vertical motion),

The total cost for a leg $e$ can then be computed using relevant change of variable.

For non vertical displacement, the cost due to a pair of features $\left(f_{i}, f_{j}\right)$ is the integral of a rational function:

$K_{i j}(x)=\frac{\left[\left(x-x^{i}\right)\left(y(x)-y^{j}\right)-\left(x-x^{j}\right)\left(y(x)-y^{i}\right)\right]^{2}}{p_{i}(x) p_{j}(x)}$.

where $p_{l}(x)=\left(x-x^{l}\right)^{2}+\left(y(x)-y^{l}\right)^{2} \triangleq a_{l} x^{2}+$ $b_{l} x+c_{l}, l \in\{j, i\}$ is the respective square range of $f_{i}, f_{j}$ to the sensor. Therefore, these polynomials are irreducible whatever the sensor position in $\mathcal{D} \backslash$ $\left\{f_{i}, f_{j}\right\}$. We can rewrite

$$
K_{i j}(x)=\frac{\left(A_{i j} x+B_{i j}\right)^{2}}{p_{i}(x) p_{j}(x)} .
$$

So, we have to compute:

$$
c_{i j}^{n v}(e) \propto \int_{x_{-}^{i j}}^{x_{+}^{i j}} K_{i j}(x) \mathrm{dx} .
$$

which can be done with a relevant partial expansion of the rational function. Nevertheless, we have to pay attention to the position of the leg relatively to the features.

case (1) $e$ is on the perpendicular bisector of

$\left[f_{i} f_{j}\right]$, then $p_{j}(x)=p_{i}(x), \forall x$ and

$$
\frac{\left(A_{i j} x+B_{i j}\right)^{2}}{p_{i}(x) p_{j}(x)}=\frac{r_{1} x+s_{1}}{p_{i}(x)}+\frac{r_{2} x+s_{2}}{p_{i}^{2}(x)} .
$$

case $(2) e$ is not on the perpendicular bisector of $\left[f_{i} f_{j}\right]$, then

$$
\frac{\left(A_{i j} x+B_{i j}\right)^{2}}{p_{i}(x) p_{j}(x)}=\frac{r_{1} x+s_{1}}{p_{i}(x)}+\frac{r_{2} x+s_{2}}{p_{j}(x)} .
$$

Identification of the numerators yields in both cases to a linear system to deduce $\chi=$ $\left[r_{1} r_{2} s_{1} s_{2}\right]^{*}$,

$$
\begin{gathered}
M_{i j}^{(c)} \chi=\mathcal{B}_{i j}, \text { for cases } \mathrm{c}=1,2 \quad(24) \\
M_{i j}^{(1)}=\left(\begin{array}{cccc}
a_{i} & 0 & 0 & 0 \\
b_{i} & 0 & a_{i} & 0 \\
c_{i} & 1 & b_{i} & 0 \\
0 & 0 & c_{i} & 1
\end{array}\right), \mathcal{B}_{i j}=\left(\begin{array}{c}
0 \\
A_{i j}^{2} \\
2 A_{i j} B_{i j} \\
B_{i j}^{2}
\end{array}\right)
\end{gathered}
$$

and

$$
M_{i j}^{(2)}=\left(\begin{array}{cccc}
a_{i} & a_{j} & 0 & 0 \\
b_{i} & b_{j} & a_{i} & a_{j} \\
c_{i} & c_{j} & b_{i} & b_{j} \\
0 & 0 & c_{i} & c_{j}
\end{array}\right)
$$

For vertical displacements, it is more appropriate to consider integration with the variable $y$. The same reasoning leads to the integration of a rational function to get the cost expression

$$
c_{i j}^{v}(e) \propto \int_{y_{-}^{i j}}^{y_{+}^{i j}} K_{i j}(y) \mathrm{dy} .
$$

\subsubsection{Closed form expression for the cost}

Whatever the leg orientation, we have to deals with the computation of integrals of the form $(n \in$ $\{1,2\}, l \in\{i, j\})$ :

$$
H^{(n)}\left(l, u, v, x_{-}, x+\right)=\int_{x_{-}}^{x_{+}} \frac{u x+v}{\left(a x^{2}+b x+c\right)^{n}} \mathrm{dx}
$$


Using specific changes of variable and classic primitives, the closed form expression for the cost (21), (27) can be derived. For instance,

$$
\begin{aligned}
& H^{(1)}\left(l, u, v, x_{-}, x+\right)=\nu_{l}^{(1)} \ln \left(\frac{\left|p_{l}\left(x_{+}\right)\right|}{\left|p_{l}\left(x_{-}\right)\right|}\right)+ \\
& \lambda_{l}^{(1)}\left(\tan ^{-1}\left(q_{l}\left(x_{+}+\frac{b_{l}}{2 a_{l}}\right)\right)-\tan ^{-1}\left(q_{l}\left(x_{-}+\frac{b_{l}}{2 a_{l}}\right)\right)\right) \\
& \text { where } q_{l}=\sqrt{\frac{4 a_{l}^{2}}{4 a_{l} c_{l}-b_{l}^{2}}}, \nu_{l}^{(1)}=\frac{u}{2 a_{l}} \text { and } \\
& \lambda_{l}^{(1)}=\frac{2 v a_{l}-u b_{l}}{2 a_{l}^{2}} q_{l} .
\end{aligned}
$$

The expressions of the costs are finally

$c_{i j}(e)=H^{(1)}\left(i, r_{1}, s_{1}, x_{-}^{i j}, x_{+}^{i j}\right)+H^{(n)}\left(j, r_{2}, s_{2}, x_{-}^{i j}, x_{+}^{i j}\right)$

where $n \in\{1,2\}$ depends on the leg orientation according to $\left[f_{i}, f_{j}\right]$. Given the contribution of each visible pair of features, the complete cost of the leg is given by $c(e)=\sum_{i, j} c_{i j}(e)$ Therefore, the cost associated to a path $\tau=\left\{e_{1}, \cdots, e_{n}\right\}$ of length $n=n_{\tau}-1$ is $c(\tau)=\sum_{i=1}^{n} c\left(e_{i}\right)$. The optimization can then be solved via dynamic programming.

\section{EXPERIMENT}

In this experiment, we consider an embedded map composed of ten features organised on the border of $\mathcal{D}=[0 ; 200 ; 0 ; 200]$. The sensor FOV is characterized by a maximum range detection $R_{\max }=70 \mathrm{~m}$ and a half aperture angle $D_{m}=120$ deg.. Moreover, the authorized difference angle between two following time steps must be bounded by $\pi / 4$ and the path length smaller than $l_{\max }=98$ legs from $q_{s}=(20 ; 20)$ to $q_{t}=$ $(170 ; 20)$. The grid resolutions are $\delta \mathrm{x}=\delta \mathrm{y}=10$. The algorithm seems to behave well. The sensor

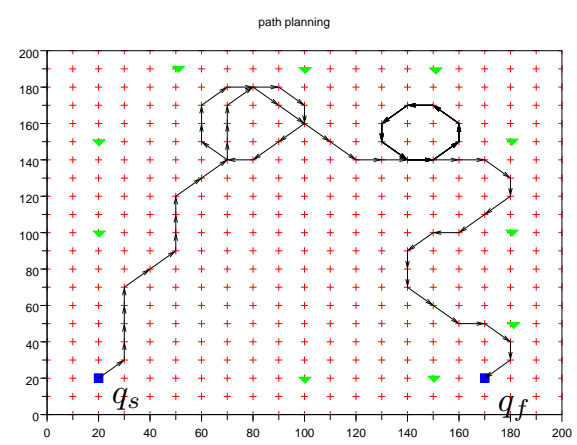

Figure 3: Optimal path, features(green), $q_{s}$ and $q_{f}$ (blue). moves in order to be as soon as possible on the perpendicular bisector of pairs of features and to increase the number of visible pairs. The proposed path allows to provide better triangulation conditions which improves the estimation process. Moreover some interesting behaviour like cycles can also be observed.

\section{CONCLUSIONS AND PERSPECTIVES}

In this paper, we introduced a path planning algorithm for map based localization. First of all, we derived an information gain as the determinant of the Fisher Information Matrix adapted to multiple features. A geometric interpretation of this measure was made. Then, to determine the optimal path, we considered the integral cost of this function. It is important to notice that the cost computation take into account the sensor field of view model. Finally, we applied the approach on a scenario and illustrate the behaviour of the algorithm. We detailed the approach for only the first part of the total cost, but it can be generalized to the others. Now, we plan to take into account noisy feature positions which will yields to a path planning problem with uncertain cost. Then, the next challenge is to find optimal paths which tackle also those uncertainties on the given map.

\section{REFERENCES}

Celeste, F., Dambreville, F., and Le Cadre, J.P. (2007). Optimal strategies based on the cross entropy method. In Fusion 2007, Quebec (Canada).

Gu, G., Chandler, P., Schumacher, C., Sparks, A., and Pachter, M. (2006). Optimal cooperating sensing using a team of uavs. IEEE Trans. on Aerospace and Electronic Systems, 42(4).

Paris, S. and Le Cadre, J.-P. (2002). Trajectory planning for terrain-aided navigation. In Fusion 2002.

S. Thrun, D. Fox, W. B. and Dellaert, F. (2000). Robust monte carlo localization for mobile robots. Artificial Intelligence, 28(1-2):99-141.

Thrun, S., Burgard, W., and Fox, D. (2005). Probabilistic Robotics. MIT press.

Van Trees, H. (1968). Detection, Estimation and Modulation Theory. New York Wiley. 